Article

\title{
Machine Learning for Quantitative Finance Applications: A Survey
}

\author{
Francesco Rundo ${ }^{1, *(\mathbb{D}}$, Francesca Trenta ${ }^{2} \mathbb{C}$, Agatino Luigi di Stallo ${ }^{3}$ and Sebastiano Battiato ${ }^{2} \mathbb{C}$ \\ 1 STMicroelectronics Srl-ADG Central R\&D, 95121 Catania, Italy \\ 2 IPLAB-Department of Mathematics and Computer Science, University of Catania, 95121 Catania, Italy; \\ francesca.trenta@unict.it (F.T.); battiato@dmi.unict.it (S.B.) \\ 3 GIURIMATICA Lab, Department of Applied Mathematics and LawTech, 97100 Ragusa, Italy; \\ distallo@distallo.it \\ * Correspondence: francesco.rundo@st.com; Tel.: +39-095-740-4566
}

Received: 27 November 2019; Accepted: 15 December 2019; Published: 17 December 2019

check for updates

Featured Application: The described approaches can be used in various applications in the field of quantitative finance from HFT trading systems to financial portfolio allocation and optimization systems, etc.

\begin{abstract}
The analysis of financial data represents a challenge that researchers had to deal with. The rethinking of the basis of financial markets has led to an urgent demand for developing innovative models to understand financial assets. In the past few decades, researchers have proposed several systems based on traditional approaches, such as autoregressive integrated moving average (ARIMA) and the exponential smoothing model, in order to devise an accurate data representation. Despite their efficacy, the existing works face some drawbacks due to poor performance when managing a large amount of data with intrinsic complexity, high dimensionality and casual dynamicity. Furthermore, these approaches are not suitable for understanding hidden relationships (dependencies) between data. This paper proposes a review of some of the most significant works providing an exhaustive overview of recent machine learning (ML) techniques in the field of quantitative finance showing that these methods outperform traditional approaches. Finally, the paper also presents comparative studies about the effectiveness of several ML-based systems.
\end{abstract}

Keywords: machine learning; time-series; financial domain

\section{Introduction}

In recent years there has been increasing interest in predicting the future behavior of complex systems by involving a temporal component [1]. Researchers have investigated this problem modelling a convenient representation for financial data, the so-called time series (i.e., numerical data points observed sequentially through time). Previous studies have highlighted the difficulty studying financial time series accurately due to their non-linear and non-stationary patterns. In [2] the authors presented a comparative study in order to demonstrate the inadequacy of classical approaches in capturing the evolution of real-time time series. Furthermore, they discussed the advantages of applying machine learning (ML) techniques in the field of quantitative finance. Despite achieving effective outcomes, classical approaches have been widely employed in stationary systems, which represent an approximation of the complex real-world observations.

The progressive automatization of certain processes and rapid development in technology has led to the use of ML-based methods in several fields, including the financial one. Despite skepticism about the effectiveness of these approaches, researchers have proven that one of the main benefits 
of ML solutions is to analyze a large amount of data in a short period of time with greater accuracy and effectiveness. Due to unclear dependencies within data, identifying significant information from irrelevant information is a very difficult task that can be tackled properly by ML-based systems.

The remainder of this paper is organized as follows. Section 2 describes how the research investigation has been conducted. Section 3 introduces main differences between two principal approaches in which surveyed studies fall into. In Section 4, we discuss the performance of auto-regressive models developed for financial market applications. Section 5 provides a description of studies based on Machine Learning algorithm, selected for our review. In addition, we discuss a class of deep learning algorithm, which achieved impressive results in time series forecast. Section 6 provides an exhaustive comparison between ML-based approaches and traditional models (such as autoregressive integrated moving average (ARIMA), generalized autoregressive conditional heteroskedasticity (GARCH), etc.). Section 7 devises some of significant hybrid approaches to identify the effective combination for improving accuracy in time-series forecasting. Section 8 lists existing work in the area of sentiment analysis. Section 9 illustrates reinforcement learning and quantum finance approaches applied in financial domain. Section 10 illustrates comparison between traditional and ML-based approaches. In Section 11, we report the final considerations of this paper.

\section{Research Methodology}

In this survey, we selected studies and/or research works based on the ML-based approach or classical method in order to analyze time series problem in financial domain.

In this paper, we shed light on the promising results achieved by machine learning approaches for time-series forecasting in the financial sector. Unlike relevant existing review articles [3], this survey not only focuses on summarizing several approaches suitable for solving financial market problems, but also compares ML-based approaches and traditional ones in order to discuss which method could be more effective considering the current financial scenario.

Also, we provided results of selected studies in order to highlight the better overall performance of ML-based systems over traditional approaches.

\section{Technical and Fundamental Analysis}

In the financial field we can distinguish two different approaches: Technical and Fundamental Analysis. The goal is to identify patterns in order to predict time-series movements and improve accuracy. The technical approach is based on analyzing stock market considering previous observed patterns with the aim to determine future time series values. In fundamental analysis (FA), the basic idea focuses on evaluating intrinsic value (i.e., perceived or calculated value of a stock or a company). Technical analysis (TA) gained a considerable attention due to the efficient market hypothesis which claims that stock movements are not a stochastic process but reveal repeated patterns over time. In fact, this approach is based on studying prices movements through analyzing historical data. Despite being a basis for several financial applications, TA presents cons related to the fact that market is highly variable. Although the use of indicators parameters and tools to achieve a high accuracy, using real data could affect the overall performance in negative perspective. On the other hand, FA consists of studying factors which may affect the future trend. The main problem regards the fact that it incurs considerable computational cost due to the poor result in performing rapid decision.

The proposed work illustrated some of the main methods that fall into each category of analysis in order to define cons and pros of each methods and evaluate what algorithm is convenient to use according to the current scenario.

\section{Autoregressive Models}

A considerable amount of literature has examined the most promising techniques for time series forecasting. The outcomes have highlighted that these approaches could be subdivided into two separate macro-categories, which include statistical and machine-learning methods. 
The ARIMA model represents a generalization of the auto-regressive moving average (ARMA) model, suitable to describe non-stationary time-series. More specifically, the main benefit in using the ARIMA model is to transform a non-stationary series into a series without seasonality or trend, by applying finite differencing of data points [4].

By definition, a time series is stationary if its statistical properties are all constant over time. If a stationary series has no trend, its variations around its mean have a constant amplitude. Furthermore, the time-series autocorrelations remain constant over time. Based on these assumptions, a time series of this form can be considered as a combination of signal and noise. An ARIMA model manages the signal by separating it from the noise. After removing noise from the signal, the output of ARIMA model is the signal step-ahead to obtain forecasts. In order to select an appropriate model to improve time-series forecasting, the Box-Jenkins model [4] has been developed to determine best ARIMA model to fit to a given time-series.

The Box-Jenkins model has gained momentum in forecasting time-series not only in financial sector but also to improve prediction of future values of a time-series considering electricity prices or traffic data observations $[5,6]$.

In order to demonstrate the effectiveness of the ARIMA model, we surveyed a set of works where the ARIMA process was applied to perform stock price forecasting.

In [7] the authors investigated the problem to produce accurate prediction of stock market time series data by using ARIMA and the GARCH model. However, they indicate that a major class of works in literature used these classical approaches for solving a one-step ahead forecasting problems. In the case of a multi-step or N-step ahead prediction, the aforementioned model does not perform accurately. In particular, authors have been concerned about the decrease of accuracy and lack of maintenance of data trend or dynamics. In order to overcome these limitations, a linear hybrid model combining ARIMA and GARCH was proposed. First, they used a simple moving average filter in order to decompose the stock-market time series into two different series. One of these data streams is modeled by using ARIMA and GARCH model respectively. Finally, the outcomes from both models are then combined to perform the values predictions. Results have shown that the proposed hybrid model is suitable for multi-step ahead forecasting of high-volatile time-series.

In [8] the authors have applied a statistical model based on ARIMA to the New York Stock Exchange (NYSE) and Nigeria Stock Exchange (NSE) for predicting future daily stock prices. The experimental results showed that the model has reached satisfactory results for both stock indexes.

Despite preserving the data trend and obtaining good predictions in terms of accuracy, the ARIMA model has definite limitations when dealing with financial time series data which present asymmetries, irregular time intervals and high/low volatility. In fact, the main assumption of the ARIMA model regards constant variance. Considering this fact, an integrated model represents a suitable solution when dealing to data which reveal a non-complex structure.

Since we have shed light to the limitation of statistical models for the analyzed time series, the next step consists in reviewing models of the machine-learning family which achieve effective results for financial data prediction. Table 1 summarizes surveyed studies in this section.

Table 1. Summary of studies based on auto-regressive models. ARIMA: autoregressive integrated moving average. GARCH: generalized autoregressive conditional heteroskedasticity.

\begin{tabular}{cccc}
\hline Article & Techniques & Main Goal & Dataset \\
\hline Babu et al. (2014) [7] & $\begin{array}{c}\text { Hybrid ARIMA-GARCH } \\
\text { model }\end{array}$ & $\begin{array}{c}\text { Multi-step } \\
\text { ahead forecasting }\end{array}$ & NSE India data \\
\hline Adebiyi et al. (2014) [8] & ARIMA model & Stock price prediction & $\begin{array}{c}\text { Nokia stock index/Zenith } \\
\text { bank stock index }\end{array}$ \\
\hline
\end{tabular}




\section{Machine-Learning Approaches}

\subsection{Support Vector Machine (SVM)}

Support vector machine (SVM) is a technique of Machine Learning for data classification. The objective of SVM is to find a separation hyperplane to correctly classify data with the maximum margin [9]. The SVM finds a decision function which maximizes the margin between classes. SVM is a supervised classification technique; this means that there are two main steps to be performed. First, a set of labelled data (training data) is provided to the SVM. During this step the algorithms performs a mathematical optimization based on the labelled data. The train examples that limit the maximum margin defined by the SVM during the training are called support vectors.

A significant work based on the SVM model is [10], in which the authors aimed to develop an efficient system to gain high profits by analyzing stock markets. Specifically, the authors employed the SVM model to select the stock that exceed the percentage return of the market only selecting stocks that outperform it. The results confirmed the effectiveness of the proposed SVM, in fact the selected range of stocks reported a total return of $208 \%$ over 5 years.

The work developed by Cao et al. [11] was motivated by the fact that SVM achieved consistent results for forecasting financial time series. This document provides a comparison between SVM, a retro-propagation multilayer neural network $(\mathrm{BP})$ and a regularized radial base function (RBF) neural network. These models have been evaluated by taking as input five datasets listed in the Chicago Mercantile Market. Results showed that SVM excels and achieves effective results considering both adaptive and free parameters.

Nayak et al. [12] presented a framework which combines the SVM and K-approach (KNN) for predicting Indian stock indices. Specifically, the authors applied the proposed model by using different SVM kernel functions for estimating profits and losses. In order to improve the stock value prediction, the author used the output, computed by SVM, to calculate the best neighbor from a training set. Furthermore, they used two indices, the Bombay Stock Exchange (BSE Sensex) and CNX Nifty, to evaluate the proposed model, taking advantages of closing price forecast, volatility and stock market momentum related to data. To provide a complete study, the authors compared the hybrid model with Functional Link Interval Type 2 Fuzzy Neural System (FLIT2NS) and Computationally Efficient Functional Link Artificial Neural Networks (CEFLANN) by analyzing mean squared error (MSE) results. Despite significantly complex procedure to update weight, the developed SVM-KNN hybrid model is relatively suitable for solving financial prediction problems.

In [13], the authors explained a new approach called the state box method which consists of selecting a group of stock points and subdividing them into three different boxes. Each box refers to a category that indicates the stock status. In this approach, the authors implemented a new method incorporating the AdaBoost algorithm, the probabilistic vector support machine (PSVM) and the genetic algorithm (GA) with the aim to improve the classification of status boxes. Each box includes a limited number of points which indicates the share price trend over a specific time period. The authors investigated the effectiveness of the proposed model by selecting a group of shares listed in the Shenzhen Stock Exchange (SZSE) and the National Association of Securities Dealers Automated Quotations (NASDAQ) as inputs. By analyzing the results, the authors confirm that ensemble classifier achieves remarkable results and outperforms other approaches based on using only the PSVM or the back-propagation neural network (BPN).

One of the major issues related to financial field is the limitation in learning the pattern because of high complexity and strong non-linear trend of stock data. In order to avoid inconsistent and unpredictable performance on noisy data, Support Vector Machine has been proposed to overcome limitations of classical approaches. Recently, SVM was not only applied for pattern recognition problems but also to solve non-linear regression problems. SVM implements the so-called structural risk minimization principle (SRM) which is an inductive principle used for model selection based on balancing the model's complexity against its success at fitting the training data. In fact, the main benefit 
of SVM model is due to the fact it achieves higher generalization performance than traditional neural network models. From the results reported in this section, it can be observed that the performance of SVM exceeds the overall performance of classical neural networks approaches. In particular, the superior performance is guaranteed by the fact that SVM implements the SRM principle which is based on minimizing upper bound of the generalization error instead of minimizing the training error. This eventually leads to better generalization than other ML-approaches (such as the BP neural network). Also, advantages in applying SVM model not only includes a major improvement in terms of generalization of neural networks performance, but also successfully overcome the defeats of other models. Unlike existing ML models, SVM present a finite number of controlling parameters, prevents overfitting problem and reaches convergence faster.

In Table 2, we report surveyed studies based on applying SVM model.

Table 2. Summary of studies based on the support vector machine (SVM) model. KNN: K-nearest neighbors.

\begin{tabular}{cccc}
\hline Article & Techniques & Main Goal & Dataset \\
\hline $\begin{array}{c}\text { Fan et al. } \\
(2001) \text { [10] }\end{array}$ & Support Vector Machine & Stock selection & $\begin{array}{c}\text { Australian Stock Exchange } \\
(1992-2000)\end{array}$ \\
\hline $\begin{array}{c}\text { Cao et al. } \\
(2003) \text { [11] }\end{array}$ & Support Vector Machine & Forecasting & Chicago Mercantile Market \\
\hline $\begin{array}{c}\text { Nayak et al. } \\
(2015) \text { [12] }\end{array}$ & Hybrid SVM-KNN model & Stock market prediction & Indian stock market indices \\
\hline $\begin{array}{c}\text { Zhang et al. } \\
(2016)[13]\end{array}$ & $\begin{array}{c}\text { Status box method (Hybrid AdaBoost } \\
\text { algorithm, genetic algorithm and } \\
\text { probabilistic SVM) }\end{array}$ & Stock trend prediction & $\begin{array}{r}\text { 20 shares from SZSE/ } \\
\text { 16 shares from NASDAQ }\end{array}$ \\
\hline
\end{tabular}

\subsection{Deep Learning}

Deep learning (DL) is a part of the machine-learning methods based on using data to train a model to make predictions from new data. In general, DL methods allow to perform difficult task without human involvement. In particular, DL methods represent an effective solution in solving financial market problems which are different from typical deep learning applications. For example, selecting a stock that is likely to perform well in future could represent a challenging task for a human. For this reason, the tools of deep learning may be useful in such selection problems, because deep learning techniques represent the best solution to compute any function mapping data (which include returns, economic data, accounting date, etc.) into the value of the return.

This document [14] deals with stock market index forecasting. The experiments were carried out by considering two indices, namely CNX Nifty and S\&P Bombay Stock Exchange (BSE) Sensex of the Indian stock markets. The main contribution of this work is the development of a two-phase fusion approach which involves the use of support vector regression (SVR) in the first phase. The second stage defined three different combination of SVR with other models including an artificial neural network (ANN), random forest (RF) and SVR. Thus, the authors provide a comparison between the resulting prediction models and single models ANN, RF and SVR for estimating the accuracy in forecasting time series 1-10, 15 and 30 days in advance.

The authors of [15] presented a long short-term memory (LSTM) neural networks for modeling and predicting the return of Chinese shares. Furthermore, they compared their LSTM model with random forecasting method in order to verify the applicability and superiority of the proposed model. The input data of the model are data related to Chinese stock market. In order to evaluate the proposed model, the authors considered 30-day sequences of historical data with 10 learning features and labeling functions of the earnings rate in 3 days. Results confirmed the superior performance of LSTM over random forecasting method and its ability to improve the accuracy related to stock returns forecasting.

In this document [16], the authors provided an innovative model called the bat-neural network multi-agent system (BNNMAS) aimed to perform stock prices prediction. In particular, the goal of the 
proposed method is to predict eight years of Deutscher Aktienindex (DAX) stock indices. Furthermore, the results of BNNMAS have been compared with the results of the genetic algorithm-neural network (GANN) and teh general regression neural network (GRNN). Efforts have demonstrated the power of BNNMAS in predicting stock prices in the long term accurately.

In [17] the authors investigated the problem of applying LSTM for financial time series forecasting. They proposed a cutting-edge technique in order to distribute LSTM for predicting off-directional movements. Stocks of the S\&P 500 from 1992 to 2015 have been used as input data to validate the proposed model. The results not only reveal that LSTM performs better without taking advantage of classification methods such as random forest or deep neural network (DNN) but also shedding light on the common pattern of securities presents high volatility and a short-term inversion return profile.

In Table 3, we summarize surveyed studies based on DL models.

\subsection{Recurrent Neural Network (RNN) for Financial Forecasting}

Recurrent Neural Networks (RNN) are a class of neural networks which uses internal memory to process arbitrary sequence of inputs. Recurrent networks differ from feedforward networks by taking as their input not just the current input example they analyze, but also what they have perceived previously in time. In fact, recurrent networks preserve sequential information through a hidden state which allows them to store information about the past efficiently. On the other hand, feedforward networks transform input examples into an output. For example, considering a supervised learning problem, the output would be a label, a name applied to the input.

A significant variant of RNN is LSTM which represents a well suitable solution in regression problem such as forecasting time series. Considering their ability to capture hidden nonlinear correlations between input data, the model was applied successfully to increase the accuracy forecast. In this section, we provide a list of works based on using the LSTM/RNN model to solve financial time-series forecasting.

LSTM architecture has proven to be stable and powerful for modeling long-range dependencies in various previous studies [18-20]. The major benefit of LSTM is the ability to store useful information by using its memory cell. In fact, LSTM represents a considerable improvement of the classic RNN model because not only is it able to evaluate the hidden non-linear correlations between input data but also it takes advantages of LSTM main unit and "gates" to prevent the so called vanishing gradient problem which represents a drawback in RNN model. 
Table 3. Summary of studies based on machine-learning (ML)/deep-learning (DL) models. ANN: artificial neural network. SVR: support vector regression. RF: Random Forest.

\begin{tabular}{|c|c|c|c|}
\hline Article & Techniques & Main Goal & Dataset \\
\hline Patel et al. (2014) [14] & $\begin{array}{l}\text { Fusion approach involving ANN, } \\
\text { RF and SVR models }\end{array}$ & Stock prices prediction & Indian Stock market indices \\
\hline Chen et al. (2015) [15] & LSTM model & Stock returns prediction & China Stock Market data \\
\hline $\begin{array}{l}\text { Hafezi et al. } \\
(2015)[16]\end{array}$ & $\begin{array}{c}\text { bat-neural network } \\
\text { multi-agent system (BNNMAS) }\end{array}$ & Stock price prediction & DAX stock price \\
\hline $\begin{array}{l}\text { Fischer et al. } \\
\text { (2017) [17] }\end{array}$ & LSTM model & $\begin{array}{l}\text { Large-scale financial } \\
\text { market prediction }\end{array}$ & $\begin{array}{l}\text { S\&P 500 } \\
(1992-2015)\end{array}$ \\
\hline $\begin{array}{l}\text { Yümlü et al. } \\
\text { (2005) [21] }\end{array}$ & $\begin{array}{l}\text { Global, recurrent and } \\
\text { smoothed-piecewise neural models }\end{array}$ & Financial time series prediction & $\begin{array}{l}\text { Istanbul Stock Exchange } \\
\quad(1990-2002)\end{array}$ \\
\hline $\begin{array}{l}\text { Selvin et al. (2017) } \\
\text { [22] }\end{array}$ & $\begin{array}{l}\text { LSTM-RNN-CNN sliding } \\
\text { window-ARIMA }\end{array}$ & Short term future prediction & $\begin{array}{l}\text { NSE listed companies' data } \\
(2014-2015)\end{array}$ \\
\hline $\begin{array}{l}\text { Samarawickrama et al. } \\
\text { (2017) [23] }\end{array}$ & RNN model & Daily stock prices prediction & Sri Lankan Stock Exchange \\
\hline $\begin{array}{l}\text { Siami-Namini et al. } \\
\text { (2018) [24] }\end{array}$ & LSTM vs. ARIMA & Comparison & N225, IXIC, HIS, GSPC, DJ \\
\hline $\begin{array}{l}\text { Rundo et al. } \\
\text { (2019) [25] }\end{array}$ & LSTM model & Forecasting & $\begin{array}{c}\text { ENEL.MI } \\
\text { ISP.MI } \\
\text { UCG.MI } \\
\text { CVAL.MI } \\
\text { MPS.MI }\end{array}$ \\
\hline $\begin{array}{l}\text { Rundo et al. } \\
\text { (2019) [26] }\end{array}$ & $\begin{array}{l}\text { Grid algorithmic trading system } \\
\text { (Non-linear Regression Network) }\end{array}$ & Forecasting & FX market (EUR/USD) \\
\hline
\end{tabular}

A significant work that provides a comparative study on evaluating the performance of three different architectures is [21], in which the authors compare a global, feedback and smoothed-wise model in order to solve the financial forecast problem. The three models include a multilayer perceptron (MLP) feedback model by a RNN and smoothed-piecewise model by a "mixture of experts" (MoE) structure. The evaluation of the aforementioned models is performed considering 12 years (from 1990 to 2002) of the Istanbul Stock Exchange (ISE) index (XU100). Furthermore, the authors implemented an exponential generalized autoregressive conditional heteroscedastic (EGARCH) model aiming to provide an exhaustive comparison based on estimating index return series such as hit rate (HR), positive hit rate $(\mathrm{HR}+)$, negative hit rate (HR-), MSE, mean absolute error (MAE) and correlation. The analysis of experiment results has highlighted that smoothed-piecewise model outperforms the other ones, including EGARCH model.

Selvin et al. [22] investigated how a DL architecture could be applied to predict stock index movement. Contrary to existing methods which are based on linear autoregressive (AR), moving average (MA), ARIMA and non-linear algorithms, the proposed approach is based on three different neural networks (LSTM, RNN and convolutional neural network (CNN)) to predict National Stock Exchange of India (NSE)-listed companies. In order to evaluate the proposed methods, the authors defined a sliding window strategy which includes overlapped data referring to minute wise data. The results of error percentage value confirm that $\mathrm{CNN}$ is able to capture the dynamical change of the data compared to other models that present a lack of accuracy.

Samarawickrama et al. [23] proposed the use of RNN to improve the accuracy of financial time series prediction. Closing, High and Low prices of selected listed companies of Colombo Stock Exchange were analyzed in order to predict future prices considering the past two days values. In this approach, the comparison involved feedforward, simple recurrent neural network (SRNN), gated recurrent unit (GRU) and LSTM architecture and make comparison between them. The prediction results showed that structural recurrent neural network (SRNN) and LSTM neural networks performed high accuracy contrary to feedforward networks. Despite the effectiveness of the two proposed 
models, they present some drawbacks in different contexts whereas feedforward present a lower error. To conclude, it has been noted that GRU networks do not perform stock price forecasting accurately.

The work proposed by Siami-Namini et al. [24] conducted a comparative study with the aim of investigating if ML based approaches can be superior to traditional methods in terms of accuracy. In this work, the authors compared a LSTM, a deep-learning architecture, with the ARIMA model which falls into the class of statistical models. In this approach, historical monthly financial time series from Jan 1985 to Aug 2018 from the Yahoo finance Web have been considered as input of the proposed models in order to predict the trend of the stock exchanges. The experiment results reported that LSTM, compared to the ARIMA model, provided the best overall performance, confirmed by RMSE values.

Rundo et al. [25] proposed an innovative LSTM-based framework in order to perform careful stock price prediction. The proposed approach includes two pipelines: the first is defined to perform Trend prediction and the second is developed to predict stock price values. Regarding stock close price prediction, the proposed system is based on the usage of LSTM together with mathematical price correction approach performed by Markov statistical model. Results revealed that the proposed framework is able to perform stock price forecasting accurately. Furthermore, the proposed method outperforms statistical model such as SMA, in terms of accuracy.

In [26], the authors presented an innovative framework composed by a non-linear regression network which performs close price forecasting. The output, computed by Regression Network, is fed to a trend classifier block which estimates the most probable short-term financial trend. After organizing the trading grid by analyzing the current state of trading, the block called the basket equity system manager evaluates the financial exposure of the trading grid.

Despite demonstrating high accuracy in predicting the next lags of time series, Integrated model represents an inappropriate solution considering the newly developed deep learning-based algorithms for forecasting time series data.

In particular, the surveyed studies conducted and reported in this article show that deep learning-based algorithms such as LSTM outperform traditional-based algorithms such as the ARIMA model. More specifically, the average reduction in error rates obtained by LSTM present a higher percentage in term of accuracy when compared to auto-regressive models indicating the superiority of LSTM to statistical models.

\section{Comparisons}

In literature, there are a huge number of papers which provide comparison between the AR model with ARIMA methodology or BPANN aiming to define the suitable model for time-series forecasting. Other related works have also analyzed the performance of SVMs and the traditional back propagation (BP) model that seem to provide a better overall performance of these models compared to AR models. In [27], the authors investigated the stock market indices forecasting applying a GARCH model in order to compare it with standard BP. Defining a proper strategy should be provided to fit an adequate model for the time series prediction. In the past few decades, several works have applied ARMA approach for univariate time series forecasting. A time series represents a process where observations are sequences of equally spaced points in time. But in financial domain, time series present large volatility and non-linearity which are difficult to process by classical methods which their assumptions are based on linearity. Several ML techniques were proposed to overcome some drawbacks of classical methods by taking advantage of their ability to capture hidden patterns between input and output data. The authors analyzed the performance of ARMA, GARCH and BP models in predicting four stock market values. The results reported that GARCH and BP models offer a performance superior to the ARMA model by using deviation performance as a criterion. However, the ARMA model is extremely effective compared to other models in terms of direction and weighted direction performance criteria. Finally, the authors observed that GARCH fitting line is closer than BP one except for the Financial Times (FT) market. 
A similar comparison was approached in [28] in which the authors demonstrate that ANN can be engaged to improve financial time-series forecasting. The authors focused on applying ANN and ARIMA models to predict PT Aneka Tambang Tbk (ANTM) by using historical daily values. The authors also referred to previous works in which ANN is employed to predict stock movement with a satisfactory result. In this paper, the most interesting observation that emerges from the comparison of the two models is that ANN outperformed the auto regressive method. These experimental results provide further justification to apply ANN model for solving a time-series forecasting problem considering its ability to detect non-linear data pattern. In this paper, the authors reported as benchmark the each sum square (ESS) result achieved by ARIMA and by ANN, confirming the effectiveness of ML-based methods.

In [29], the ISE Index has been considered to perform time-series forecasting. The authors provide a complete study for estimating the overall performance of an ANN and SVM model. To evaluate both models, ten technical indicators have been employed including SMA etc. After establishing the best combination of parameters for both models, the authors plugged the data into ANN and SVM model aiming to forecasting the future values of the stock market. The experiments revealed that ANN is more effective than the SVM model. However, the authors shed light that both models present lower accuracy in forecasting stock market related to year 2001 in which the economic and financial crises had an immense adverse impact on the Turkish financial market.

In [30], the authors examine the results of ARIMA and ANN models in forecasting the NYSE stock data. By comparing all empirical results, ANN are more efficient than the statistical model, ARIMA.

According to [31], a random forest model can be engaged to predict stock market trend achieving good results. In particular, the authors compared the performance of ANN, SVM, random forest and naive Bayes using Reliance Industries and Infosys Ltd., in addition to CNX Nifty and S\&P Bombay Exchange (BSE) Sensex. The core idea is based on transforming data from continuous to discrete values of a fixed number (i.e., 10) of technical parameters. The authors have set up the conversion of the two first parameters, simple moving average (SMA) and weighted moving average (WMA), by comparing their value to current price. The trend is determined by setting the discrete value equal to -1 in case of the parameter value is lower than current price value which indicates a decreasing trend, +1 otherwise. The experimental results highlighted that ANN, random forest and SVM model present good performance by using discrete values as input. In general, the results illustrated that the model produces poor results for the trend prediction when considering continuous data. Technical indicators provide intrinsic information about the data trend, so they represent the adequate representation of data as input for each model. Thus, the model must find a relationship between the input trend and the output trend, which requires a lower level of complexity than that required when analyzing continuous data. By contrast, when continuous data are used, the model is not able to obtain information about the trend of the data which represents intrinsic information in these indicators.

Another example of work which focuses on applying artificial neural networks is [32]. A range of companies listed in the São Paulo Stock Exchange Brazilian Mercantile and Futures Exchange Bovespa (BM\&FBovespa) were analyzed with the purpose to forecast the minimum and maximum daily prices of the shares. In particular, the proposed pipeline is based on extracting relevant attributes for improving the forecast prediction. The authors tested the developed algorithm by using MAE, mean absolute percentage error (MAPE) and root mean square error (RMSE) metrics. Results showed that the model is effective.

Li et al. [33] compared the use of LSTM and SVM in predicting the price movements of stocks. The authors focused on analyzing a range of stocks including Shanghai Stock Exchange 50 (SSE 50) Index and which are affected by different volatility. The overall performance reveal that SVM model achieves significant results for all stocks including the SSE 50 which presents values that does not fluctuate dramatically. Despite the effectiveness of SVM, it has been noted that the LSTM presents consistent results in forecasting SSE 50 Index affected by high volatility. Considering the low-volatility 
stock exchanges, LSTM reported an averaged accuracy of 51.78\% which is higher than the SVM results $(50.82 \%)$.

The surveyed studies of this section are reported in Table 4.

Table 4. Summary of comparative studies. GARCH: Generalized Autoregressive Conditional Heteroskedasticity.

\begin{tabular}{|c|c|c|c|}
\hline Article & Method & Main Goal & Dataset \\
\hline $\begin{array}{l}\text { Hossain et al. } \\
\text { (2008) [27] }\end{array}$ & $\begin{array}{c}\text { GARCH vs. } \\
\text { Neural Network }\end{array}$ & Comparison & $\begin{array}{l}\text { Nikkei 225, Hang Seng, } \\
\text { FTSE } 100 \text { and DAX }\end{array}$ \\
\hline $\begin{array}{l}\text { Wijaya et al. } \\
\text { (2010) [28] }\end{array}$ & ARIMA vs. ANN & Comparison & $\begin{array}{l}\text { Indonesia Stock data } \\
\text { (ANTM) }\end{array}$ \\
\hline $\begin{array}{l}\text { Kara et al. } \\
\text { (2011) [29] }\end{array}$ & ANN and SVM model & $\begin{array}{l}\text { Stock price index } \\
\text { movement prediction }\end{array}$ & Istanbul Stock Exchange (ISE) \\
\hline $\begin{array}{l}\text { Adebiyi et al. } \\
\text { (2014) [30] }\end{array}$ & ARIMA vs. ANN & Comparison & New York Stock Exchange \\
\hline $\begin{array}{l}\text { Patel et al. } \\
\text { (2015) [31] }\end{array}$ & $\begin{array}{l}\text { ANN, SVM, RF, } \\
\text { Naïve Bayes }\end{array}$ & $\begin{array}{c}\text { Comparison } \\
\text { (direction of movement for stock) }\end{array}$ & Indian Stock Exchange \\
\hline $\begin{array}{l}\text { Laboissiere et al. } \\
\text { (2015) [32] }\end{array}$ & ANN & Forecasting & $\begin{array}{c}\text { São Paulo Stock Exchange } \\
\text { BM\&FBovespa. }\end{array}$ \\
\hline $\begin{array}{l}\text { Li et al. } \\
\text { (2017) [33] }\end{array}$ & RNN, SVM & Stock prediction & $\begin{array}{c}\text { Shanghai Stock Exchange } \\
50 \text { (SSE 50) }\end{array}$ \\
\hline
\end{tabular}

\section{Hybrid Systems}

A new hybrid approach [34] have been devised in order to forecast trend by using Taiwan stock exchange weighted stock index (abbreviated TSEWSI). The hybrid system was developed by combining a RNN model which uses features extracted by ARIMA model as input data. To improve the overall performance, neural networks have been fed with the difference of predictions sequence in order to adjust the connection weights during backpropagation training. Experiments revealed that the prediction performed by the proposed model was significant and reliable. In fact, it has the capability to predict 6 weeks ahead the market trend.

Another work that takes advantages from both linear and non-linear models is [35]. In this work, the authors developed a hybrid method involving ARIMA and ANN models. The results confirmed that the proposed model is well suited to improve forecasting accuracy. The aim of the proposed models was to predict 35 and 67-period ahead. Results reported that neither the neural network nor the ARIMA model have the capability to understand the patterns within data. But it has been noted that the hybrid model combining two models is able to reduce the overall forecasting errors for 67-period forecasting. With regard to 35-period forecasting, the ANN achieved better results.

The authors of [36] presented a new hybrid asymmetric volatility approach based on artificial neural networks. In particular, they focused on improving the forecasting ability of derivative securities prices. The benefit of using the proposed method is due to not only its ability to capture asymmetric volatility but also the ability to reduce the stochastic and non-linearity of the error term sequence.

Lee et al. [37] provides a mixture of the SVM model and hybrid feature selection method aiming to predicting the trend of stock markets. To improve the trend prediction, a hybrid selection approach has been defined as a method to select the optimal features. The experiments were carried out by performing a comparison between SVM model with F-score and supported sequential forward search (F_SSFS) feature selection methods and BPNN along with information gain, symmetrical uncertainty, and correlation-based feature selection. The final results indicated not only that SVM achieved better results than BPN in terms of stock trend prediction but also the proposed SVM incorporating F_SSFS shows a reliable level of accuracy. 
In [38], the authors present a hybrid system to forecast financial time-series. The proposed method combines an ARIMA model and fuzzy logic in order to demonstrate that proposed model could overcome data limitations of individual ARIMA model.

A reliable work based on applying hybrid method to solve stock index forecasting is [39]. Since ESM, ARIMA and BPNN models have been widely used with remarkable results to forecast time-series, this document proposed a hybrid approach involving the aforementioned models. In particular, the authors shed light on hybrid methods performs better than all three models in a single scenario in which they have been considered in the original form. Also, the proposed model achieves better results than the equal weight hybrid model (EWH), and the random walk model (RWM).

In [40] the authors have devised a hybrid system by using a genetic fuzzy system with an ANN model. The main contribution of this work is the stepwise regression analysis which has been developed to identify the factors that affect stock prices. To evaluate the overall performance of the proposed model, the experiments were carried out considering the data related to the stock of IBM and DELL corporations as well as Ryanair data, already used in previous studies. In general, it has been noted that the artificial neural networks as well as the fuzzy algorithms have been applied successfully to solve forecasting problems since they are able to obtain better results than using a single model.

In [41], the authors assembled a new hybrid approach combining the ARIMA with ANN model in order to predict financial time-series. Before applying the proposed hybrid model, the nature of financial time series has been explored in order to analyze its volatility. Numerical results showed that the hybrid model excels over the individual ARIMA and ANN models, and existing hybrid ARIMA-ANN models.

Panigrahi et al. [42] have elaborated a high-efficiency methodology combining ETS and ANN model. The proposed hybrid model takes advantages of linear as well as non-linear modeling capability. In this approach, the ETS model performs prediction of a given time series in order to capture data linear patterns. To evaluate the accuracy of predictions, the authors calculated the residual error by considering the difference between ETS predictions series and original time-series. The second stage used ETS-predictions and ANN-predictions in order to merge them. To verify the applicability and superiority of the proposed model, the authors compared this approach with some existing methods such as ARIMA, ETS, MLP and other ARIMA-ANN models. The experiments confirmed the promising results achieved by the ETS-ANN model.

In [43], the authors presented an innovative hybrid system which consists of a long short-term memory (LSTM) to forecast stock price volatility with GARCH-type models. In order to conduct the experiments, the authors used KOSPI 200 index data. The prediction performance confirmed that the proposed model yield to consistent results.

In Table 5, we reported the surveyed studies based on applying hybrid systems.

Table 5. Summary of studies based on hybrid models. IBM: International Business Machines NASDAQ: National Association of Securities Dealers Automated Quotation ESM: Exponential Smoothing Model.

\begin{tabular}{cccc}
\hline Article & Techniques & Main Goal & Dataset \\
\hline $\begin{array}{c}\text { Wang et al. } \\
(\text { (1996) [34] }\end{array}$ & $\begin{array}{c}\text { ARIMA-based Recurrent } \\
\text { Neural Network }\end{array}$ & Market trend prediction & Taiwan stock exchange \\
\hline $\begin{array}{c}\text { Zhang et al. } \\
(\text { 2003) [35] }\end{array}$ & Hybrid ARIMA-ANN model & Forecasting & $\begin{array}{c}\text { Wolf's sunspot data, Canadian lynx } \\
\text { data/British Pound/USD exchange rate data }\end{array}$ \\
\hline $\begin{array}{c}\text { Wang et al. } \\
(2009) \text { [36] }\end{array}$ & $\begin{array}{c}\text { Hybrid asymmetric } \\
\text { volatility approach } \\
\text { (Grey-GJR-GARCH) }\end{array}$ & Forecasting & Taiwan stock index \\
\hline $\begin{array}{c}\text { Ming-Chi Lee } \\
(2009) \text { [37] }\end{array}$ & $\begin{array}{c}\text { SVM with Hybrid feature } \\
\text { selection method (F_SSF) }\end{array}$ & Stock trend prediction & NASDAQ Index \\
\hline $\begin{array}{c}\text { Khashei et al. } \\
(2009) \text { [38] }\end{array}$ & $\begin{array}{c}\text { Hybrid ARIMA-ANN-Fuzzy } \\
\text { Logic model }\end{array}$ & Forecasting & $\begin{array}{c}\text { Exchange rate (US dollars/Iran Rials), } \\
\text { Gold price (Gram/US dollars), } \\
\text { Exchange rate (Euro/Iran Rials) }\end{array}$ \\
\hline
\end{tabular}


Table 5. Cont.

\begin{tabular}{cccc}
\hline Article & Techniques & Main Goal & Dataset \\
\hline $\begin{array}{c}\text { Wang et al. } \\
(2012) \text { [39] }\end{array}$ & ESM, ARIMA, BPNN & Forecasting & $\begin{array}{c}\text { Shenzhen Integrated Index (SZII), } \\
\text { Dow Jones Industrial Average Index (DJI) }\end{array}$ \\
\hline $\begin{array}{c}\text { Hadavandi et al. } \\
(2013) \text { [40] }\end{array}$ & $\begin{array}{c}\text { Hybrid Genetic Fuzzy } \\
\text { Systems-ANN model }\end{array}$ & Stock price forecasting & IBM, British Airlines, RyanAir, DELL \\
\hline $\begin{array}{c}\text { Babu et al. } \\
(2014) \text { [41] }\end{array}$ & Hybrid ARIMA-ANN model & Forecasting & $\begin{array}{c}\text { Sunspot data, electricity data, } \\
\text { L\&T stock market }\end{array}$ \\
\hline $\begin{array}{c}\text { Panigrahi et al. } \\
(2017) \text { [42] }\end{array}$ & Hybrid ETS-ANN model & Forecasting & IBM \\
\hline $\begin{array}{c}\text { Kim et al. } \\
(2018)[43]\end{array}$ & $\begin{array}{c}\text { Hybrid LSTM-multiple } \\
\text { GARCH-type model }\end{array}$ & Volatility prediction & KOSPI 200 index \\
\hline
\end{tabular}

\section{Sentiment Analysis}

As already mentioned, social media usage has increased over the last 10 years, becoming the first place to obtain news. In particular, it has been noted that social media can affect not only public opinion but also political and economic events. Many researchers have investigated how social media could affect financial market in order to gain information for forecasting financial time-series.

Satisfactory results have been achieved by analyzing textual analysis of breaking financial news. The proposed method [44] collected more than 9000 financial news articles and 10,000,000 S\&P 500 stock quotes for a period of 5 weeks. The core idea is to estimate a discrete share price by analyzing news $20 \mathrm{~min}$ after its release. Several textual representations have been used to analyze news accurately: bag of words, noun phrases and named entities. Furthermore, the authors developed a SVM-based system to perform discrete numerical prediction. The experiments demonstrated not only that Bag of Words is the representation that works better than others but also SVM is extremely accurate according to MSE values.

Another significant work in which a machine learning-based system is applied to analyze Twitter data in the financial domain is [45]. The authors suggest that the stock market trend is high correlated with public opinion. In particular, they focused on analyzing users' tweets using two different textual representations, called Word2vec [46] and N-gram [47]. The first representation was used to map each single word in a vector, the second one is used to match the corpus of a text. The experimental results confirmed the strong correlation existing between stock market trend and public opinion expressed in tweets. In Table 6, we provide a summary of surveyed studies focused on sentiment analysis.

Table 6. Summary of studies based on sentiment analysis.

\begin{tabular}{cccc}
\hline Article & Techniques & Main Goal & Dataset \\
\hline $\begin{array}{c}\text { Schumaker et al. } \\
(2009) \text { [44] }\end{array}$ & SVM & Discrete stock price estimation & S\&P 500 \\
\hline $\begin{array}{c}\text { Pagolu et al. } \\
(2016)[45]\end{array}$ & Sentiment Analysis & Stock market movements prediction & $\begin{array}{c}\text { Dow Jones Industrial } \\
\text { Average Index (DJIA) }\end{array}$ \\
\hline
\end{tabular}

\section{Reinforcement Learning (RL)}

In this section, we surveyed some considerable approaches based on reinforcement learning. Reinforcement learning refers to a machine-learning paradigm which involves an "agent" in order to perform a task. The goal of agent is to maximize a numerical reward by interacting with an unknown environment. In financial field, this emerging paradigm has gained attention due to their ability to develop portfolio construction including prediction in one integrated step. Basically, the goal of the agent is to include some constraints such as liquidity and transactions cost when a decision-making process is performed. 
In [48] the authors presented a time-driven feature-aware jointly deep reinforcement learning (RL) model (TFJ-DLR) in order to learn features representation considering noisy financial time series. Furthermore, the proposed method is developed to perform a decision-making system by taking advantage of environmental representation. Specifically, the authors selected various features of financial time series in order to summarize the useful values through analyzing historical data. Results demonstrated the robustness and effectiveness of the proposed TFJ-DLR system by using real-world financial data. In particular, the proposed method is able to increase investment returns.

A recent work is [49], in which the author implemented an algorithm based on supervised $\mathrm{DL}$ and reinforcement learning algorithm. The main goal is to forecast the short-term trend in the currency FOREX (FOReign EXchange) market with the aim to maximize the return of investment in a high-frequency trend (HFT) algorithm. Also, the author proposed a grid trading engine to perform high frequency operations. In order to validate the proposed model, the trading system has been validated over several financial years and on the EUR/USD cross. The value of return of investment $(98.23 \%)$ and reduced drawdown $(15.97 \%)$ confirmed the effectiveness of the proposed trading system. Table 7 reports surveyed articles based on RL approach.

In [50], the author proposed a model that combines dynamic programming (DP) and RL techniques. The proposed Q-Learner in the Black-Scholes (QLBS) model is developed to estimate future changes for stock market using Black-Scholes-Merton's model. After comparing fitted Q iteration to the DP model in order to evaluate the performance, the author formulated an inverse reinforcement learning (IRL) setting for the QLBS model aimed at analyzing prices and actions. Finally, the author discussed the usage of QLBS model for option portfolios.

Recently, the increasing request of developing intelligent financial systems has led to the development of trading systems which integrate quantum finance theory to solve financial problems [51,52]. The main benefit of applying quantum technology to financial problem is related to the ability of making calculations that reveal dynamic arbitrage possibilities that competitors are unable to see. Quantum computing not only could be applied to an asset management or trading problem, but it could perform trading optimization, risk profiling and prediction.

Table 7. Summary of studies based on reinforcement learning (RL) models. TFJ-DRL: Time-Driven Feature-aware Jointly Deep Reinforcement Learning.

\begin{tabular}{cccc}
\hline Article & Techniques & Main Goal & Dataset \\
\hline $\begin{array}{c}\text { Lei et al. } \\
(2020)[48]\end{array}$ & TFJ-DRL model & Financial signal representation & S\&P 500 \\
\hline $\begin{array}{c}\text { Rundo } \\
(2019) \text { [49] }\end{array}$ & $\begin{array}{c}\text { Deep Learning/ } \\
\text { Reinforcement Learning }\end{array}$ & Forecasting & EUR/USD \\
\hline $\begin{array}{c}\text { Halperin } \\
(2019)[50]\end{array}$ & $\begin{array}{c}\text { Dynamic Programming/ } \\
\text { Reinforcement Learning }\end{array}$ & Trading & Simulated stock price histories \\
\hline $\begin{array}{c}\text { Baaquie } \\
(2019)[53]\end{array}$ & Quantum Finance & Range accrual swap & 90-day Libor \\
\hline $\begin{array}{c}\text { Lee (2019) } \\
{[54]}\end{array}$ & CT2TFDNN model & Financial prediction & $\begin{array}{c}\text { Data provided by Quantum } \\
\text { Finance Forecast Center (QFFC) }\end{array}$ \\
\hline $\begin{array}{c}\text { Lee (2019) } \\
{[55]}\end{array}$ & COSMOS model & Forecasting & $\begin{array}{c}\text { Data provided by Quantum } \\
\text { Finance Forecast Center (QFFC) }\end{array}$ \\
\hline
\end{tabular}

In this context, the work of Baaquie [53] represents an innovative solution. The objective of this study [53] consists in taking advantage of quantum finance theory to price the rate range accrual swaps which refer to the exchange of one set of cash flows for another.

With the exponential growth of RL solutions, several hybrid systems based on fuzzy logic, genetic algorithms and chaotic theory have become a crucial topic in the fintech field. One of the most recent work is [54], in which the author presented a chaotic type-2 transient-fuzzy deep 
neuro-oscillatory network with retrograde signaling (CT2TFDNN) to perform financial prediction. The proposed model is intended as extension of the prior work of the same author in which a chaotic oscillatory multi-agent-based neuro-computing system (COSMOS) has been proposed [55].

In [54], the main contribution consists in providing an effective solution to preserve systems by dealing with deadlock problems which are very common in recurrent neural networks solution. Moreover, a chaotic deep neuro-oscillatory network with retrograde signaling (CDNONRS) is designed to improve time-series prediction.

\section{Discussion}

In this survey, we described some of the most promising directions adopted to solve financial problems. We started our investigation selecting the main approaches based on traditional methods, commonly used for time-series forecasting. In Table 1, we summarized studies based on ARIMA and GARCH models. First, linear models have been suggested to perform time-series forecasting taking advantage of their effectiveness and quite simple implementation. Then, non-linear models (such as ARIMA) have drawn attention due to their ability to transform non-linear data into stationary one. By comparing the results, it can be observed that surveyed methods based on traditional models, showed several weaknesses in processing a huge set of heterogenous data and identifying specific hidden patterns. This has led to an increasing demand to adopt more efficient algorithms which are able to capture hidden relationships between data, such as Machine Learning algorithms.

In recent years, several machine-learning methods have been developed for modeling financial time-series. The SVM model belongs to this category of algorithms. In SVM, the basic idea is to define an optimal hyperplane through mapping the data into higher-dimensional spaces, in which they are linearly separable.

Despite achieving remarkable results, SVM presents some problem concerning the definition of its hyper-parameters along the issue of selecting SVM training data from large datasets which could increase the time complexity and affect the overall performance of the SVM classifier in practice.

Considering these facts, other ML approaches (including ANN, LSTM and RNN) have gained a considerable uptake for time-series forecasting in last few years. One of the primary approaches was the ANN model. ANNs have been developed in order to mimic the intelligence of the human brain automatically. More specifically, ANNs try to recognize patterns in the input data attempting to provide generalized results based on their known previous knowledge. As confirmed by reported results, ANNs have largely applied in the financial domain. Their main advantage consists in not providing any a priori assumption about the data. In fact, the model is suitable to fit a given time-series by analyzing the features extracted from complex data, as opposed to traditional models, such as ARIMA.

Recently, a considerable amount of literature has investigated the use of RNN and its variants, such as LSTM, for time-series forecasting. Compared to the classical ANN model, these models achieved better results in forecasting problems due to their powerful ability to capture hidden relationships within data. More specifically, LSTM architecture has been designed to learn long-term dependencies. LSTM is able to manage the input/output data flow through its fundamental unit: the cell. A LSTM cell is composed by three different "gates", called input, forget and output gate, which establish to store or discard data by using sigmoid function, called "activation function". Also, the input and status of the cell is updated through applying the "tanh function".

Advances in natural language processing (NLP) and DL fields have brought the development of sentiment analysis approaches to transform upcoming web news, tweets and financial reports into relevant features. After extracting useful information from data in textual format, they are processed as input time series to perform forecasting.

The studies reported in this work confirmed further the effectiveness of sentiment analysis methods in predicting financial trend. 
Finally, we investigated the problem to define a profitable trading system by applying RL approaches. Thus, recent development in this field combing both DL and RL approaches taking advantage of their powerful ability to elaborate complex data.

In particular, RL approaches have been applied to deal with the prediction of medium-short term trend which represents an issue of the HFT systems. In Figure 1, we reported a timeline of strategies adopted for financial time-series modelling.

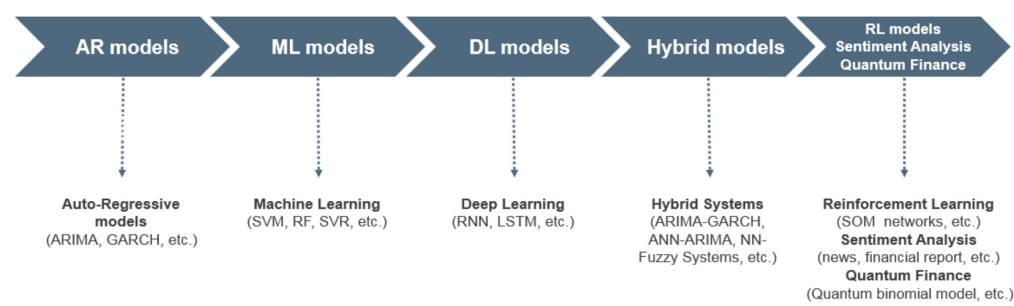

Figure 1. Timeline of adopted approaches for time-series analysis. AR: Auto-Regressive. ML: Machine Learning. DL: Deep Learning. RL: Reinforcement Learning. SVM: Support Vector Machine. RF: Random Forest. SVR: Support Vector Regression. ARIMA: Autoregressive Integrated Moving Average. GARCH: Generalized Autoregressive Conditional Heteroskedasticity. RNN: Recurrent Neural Network. LSTM: Long Short-Term Memory. SOM: Self-Organising Map.

The objective of this survey has been to discuss how machine-learning approaches outperform methods based on non-linear algorithm underlying their pros and cons. The main benefit on applying ML-based systems is to automatize trading operations and reduce computing time, ensuring to perform with high level in terms of accuracy. For this reason, we focused on ML algorithms such as SVM or random forest and DL models (LSTM or RNN). We compared some of these methods and evaluated the approaches, which obtained the best results related to time-series modeling.

Our investigation suggested that a quite considerable number of investigated studies provide an evaluation by using the following stock market data:

- Bombay SE: The Bombay Stock Exchange (BSE) is one of the largest exchanges in the world which includes around 6000 companies. A large part of these companies is also listed on the National Stock Exchange of India.

- S\&P 500: Standard \& Poor's 500 Index is a market-capitalization-weighted index of the 500 largest U.S. publicly traded companies.

- DAX indices: DAX is a stock index for German companies that trade on the Frankfurt Exchange. In order to calculate the DAX Index, an electronic trading system, called Xetra, is used. Unlike most indexes, the DAX is updated using futures prices for the next day.

- In a different twist from most indexes, the DAX is updated with futures prices for the next day, even after the main stock exchange has closed.

- Shangai stock exchange: The Shanghai Stock Exchange (SSE) represents the largest stock exchange in China. It is a non-profit organization run by the China Securities Regulatory Commission (CSRC). Stocks, funds, bonds, and derivatives are all traded on the exchange.

- NASDAQ index: The NASDAQ is the world's fast electronic stock exchange which operates through computer and telephones, as opposite to traditional method. NASDAQ lists only technology-based companies.

However, the proposed investigation highlighted the need to define a unique benchmark dataset. We observed that investigated methods conducted experiments by using different datasets. Benchmarks are important for researchers in order to measure the performance of an algorithm on a specific problem. Without the definition of a unique dataset and appropriate performance indicators, researchers cannot make a complete comparison between the proposed studies in order to select a 
suitable solution for a specific problem. Also, the majority of investigated primary studies provide different evaluation metrics for time-series forecasting.

In this work, we examined several proposed studies reporting the use of both traditional linear model and machine-learning approaches. Despite facing issues such as lack of a benchmark dataset and performance indicators, we attempted to provide a comparison between traditional and ML-based approaches, reported in this survey. Specifically, Table 8 summarizes experimental results regarding two of the investigated approaches [7,22]. The work of Babu et al. [7] proposed a hybrid system based on ARIMA and GARCH models. The authors collected data from January 2010 to January 2011 to define the first dataset (TD1) used for evaluating the performance of their model. As reported in [7], they compared results between the proposed approach and other autoregressive models such as ARIMA, GARCH etc. The corresponding errors measures (MAPE, MAE, MaxAPE and RMSE) show that the proposed approach outperforms other models. Furthermore, the authors considered SB1 shares from January 2010 to December 2010 to test the performance of proposed approach. The error performance measures (MAE, MaxAPE, etc.) confirmed that the proposed method obtains better results among others model (ARIMA and GARCH single scenario etc.). Also, the proposed hybrid system minimizes error performance. Despite achieving considerable results, autoregressive models present several limitations for stock price prediction compared to ML-based techniques.

Table 8. Summary of studies based on ML/DL models. MAPE: mean absolute percentage error. MAXAPE: maximum absolute percentage error MAE: mean absolute error. RMSE: root mean square error. NSE: Nigeria Stock Exchange.

\begin{tabular}{|c|c|c|c|c|c|c|}
\hline & Techniques & MAPE & MAXAPE & MAE & RMSE & Dataset \\
\hline \multirow{4}{*}{$\begin{array}{c}\text { Babu et al. } \\
\text { (2014) [7] }\end{array}$} & \multirow[b]{2}{*}{ Hybrid ARIMA-GARCH } & 0.1976 * & $0.4285 *$ & 0.5533 & 0.7514 & NSE (TSD1) \\
\hline & & 3.41 & 7.04 & 55.5303 & 66.1981 & NSE (TSD2) \\
\hline & \multirow[b]{2}{*}{ ARIMA } & 2.4 * & 5.3657 * & 6.3765 & 6.7195 & NSE (TSD1) \\
\hline & & 9.8 & 15.73 & 160.9963 & 169.7036 & NSE (TSD2) \\
\hline & \multicolumn{6}{|c|}{ Error Percentage } \\
\hline & \multirow{3}{*}{ RNN } & \multicolumn{4}{|c|}{3.90} & NSE (Infosys) \\
\hline & & \multicolumn{4}{|c|}{7.65} & NSE (TCS) \\
\hline & & \multicolumn{4}{|c|}{3.83} & NSE (Cipla) \\
\hline & \multirow{3}{*}{ LSTM } & \multicolumn{4}{|c|}{4.18} & NSE (Infosys) \\
\hline & & \multicolumn{4}{|c|}{7.82} & NSE (TCS) \\
\hline & & \multicolumn{4}{|c|}{3.94} & NSE (Cipla) \\
\hline \multirow{6}{*}{$\begin{array}{l}\text { Selvin al. } \\
\text { (2017) [22] }\end{array}$} & \multirow{3}{*}{$\mathrm{CNN}$} & \multicolumn{4}{|c|}{2.36} & NSE (Infosys) \\
\hline & & \multicolumn{4}{|c|}{8.96} & NSE (TCS) \\
\hline & & \multicolumn{4}{|c|}{3.63} & NSE (Cipla) \\
\hline & \multirow{3}{*}{ ARIMA } & \multicolumn{4}{|c|}{31.91} & NSE (Infosys) \\
\hline & & \multicolumn{4}{|c|}{21.16} & NSE (TCS) \\
\hline & & \multicolumn{4}{|c|}{36.53} & NSE (Cipla) \\
\hline
\end{tabular}

* The values shown in fractions instead of percentages.

In [22], the authors chose stock prices of National Stock Exchange (NSE) India data to evaluate the proposed pipeline. More specifically, they collected Infosys data for the period from July 2014 to October 2014 as training data and they used stock price for Infosys, Tata Consultancy Service (TCS) and Chemical Industrial and Pharmaceutical Laboratories (CIPLA) from October 2014 to November 2014. The reported results in Table 8 are related to Error Percentage. In particular, they compared results achieved by RNN, LSTM and CNN to ARIMA model. They observed that the ARIMA model 
present a higher error percentage value than other models, confirming that neural models are more suitable for the prediction of stock markets affected by high volatility.

In order to confirm the effectiveness of DL, we reported performance results of two surveyed works $[17,48]$ based on applying a recurrent model (LSTM) and the RL approach, respectively. The work of Fischer et al., proposed a LSTM-based model. The authors reported performance results in terms of risk characteristics considering S\&P 500 data before and after transaction costs. In Table 9, we summarized the performance results of the LSTM model. As reported in [17], LSTM shows the lowest maximum drawdown compared to other models.

In terms of annualized risk-return, LSTM achieves highest returns (0.8229) compared to random forest, DNN, etc. We reported results of [48] in which the authors applied a time-driven feature-aware jointly deep reinforcement-learning model (TFJ-DRL) to improve the financial signal representation learning. They considered stocks from S\&P 500 to evaluate the proposed model which takes advantages of Reinforcement Learning paradigm. The evaluation metrics, total profits (TP), annualized rate of return (AR), annualized Sharpe ratio (SR) and transaction times (TT), highlighted that the proposed TFJ-DRL outperforms other competitors. Also, the reported results in [48] confirmed that the proposed framework could be applied on real data stock market to achieve reliable results.

Table 9. Summaries of results achieved by a ML-based approach and a reinforcement learning (RL)-based approach considering S\&P 500.

\begin{tabular}{|c|c|c|c|c|c|c|}
\hline & Techniques & MAX DrawDown (DD) & SR p.a & Ret & n p.a & Dataset \\
\hline \multirow{3}{*}{$\begin{array}{l}\text { Fischer et al. } \\
\text { (2017) [17] }\end{array}$} & \multirow[t]{3}{*}{ LSTM } & 0.4660 & 5.8261 & \multicolumn{2}{|c|}{2.0127} & $\begin{array}{c}\text { S\&P } 500 \\
\text { (before transaction costs) }\end{array}$ \\
\hline & & 0.5233 & 2.336 & \multicolumn{2}{|c|}{0.8229} & $\begin{array}{c}\text { S\&P } 500 \\
\text { (after transaction costs) }\end{array}$ \\
\hline & & TP & $\operatorname{AR}(\%)$ & SR & TT & \\
\hline $\begin{array}{l}\text { Lei et al. } \\
(2020)[48]\end{array}$ & TFJ-DRL & 3431.87 & 20.92 & 1.09 & 609 & S\&P 500 (rising) \\
\hline
\end{tabular}

Further interesting results in the field of HFT trading algorithms and time-series forecasts, have been recently obtained by applying some deep-learning approaches based on the morphological-temporal analysis of the data initially applied in the medical and industrial sector for the study of one-dimensional aperiodic physiological signals (very close to financial time-series) [56-58]. In this perspective, as already anticipated in the work proposed by the authors in [49], the aforementioned innovative approaches are able to significantly improve both the trend forecasting capability as well as the overall performance of the developed trading system.

\section{Conclusions}

In this survey, we have reviewed some of the most promising approaches applied in financial domain. Specifically, the contribution of our work reaches multiple goals. Firstly, we defined the problems related to analyzing time-series and how traditional approaches, such as AR methods, represented an effective solution to achieve satisfactory results in financial problems. However, we provided a comparison between traditional approaches and a ML-based system in order to highlight how ML-based algorithms outperform classical approaches in terms of accuracy. Also, we demonstrated the advantages related to DL models providing an exhaustive discussion on current approaches in the RL, sentiment analysis and quantum finance fields.

Author Contributions: Conceptualization \& Investigation, F.R., F.T.; Validation, F.R., S.B.; Writing-Review and Editing, S.B. and A.L.d.S.

Funding: This research received no external funding.

Conflicts of Interest: The authors declare no conflict of interest. 


\section{References}

1. Hamilton, J.D. Time Series Analysis; Princeton University Press: Princeton, NJ, USA, 1994; Volume 2, pp. 690-696.

2. Cheng, C.; Sa-Ngasoongsong, A.; Beyca, O.; Le, T.; Yang, H.; Kong, Z.; Bukkapatnam, S.T. Time series forecasting for nonlinear and non-stationary processes: A review and comparative study. IIE Trans. 2015, 47, 1053-1071. [CrossRef]

3. Cavalcante, R.C.; Brasileiro, R.C.; Souza, V.L.; Nobrega, J.P.; Oliveira, A.L. Computational intelligence and financial markets: A survey and future directions. Expert Syst. Appl. 2016, 55, 194-211. [CrossRef]

4. Box, G.E.P.; Jenkins, G.M. Time Series Analysis: Forecasting and Control; Holden-Day: San Francisco, CA, USA, 1970.

5. Contreras, J.; Espinola, R.; Nogales, F.J.; Conejo, A.J. ARIMA models to predict next-day electricity prices. IEEE Trans. Power Syst. 2003, 18, 1014-1020. [CrossRef]

6. Williams, B.M.; Hoel, L.A. Modeling and forecasting vehicular traffic flow as a seasonal ARIMA process: Theoretical basis and empirical results. J. Transp. Eng. 2003, 129, 664-672. [CrossRef]

7. Babu, C.N.; Reddy, B.E. Selected Indian stock predictions using a hybrid ARIMA-GARCH model. In Proceedings of the 2014 International Conference on Advances in Electronics Computers and Communications, Bangalore, India, 10-11 October 2014; pp. 1-6.

8. Adebiyi, A.A.; Adewumi, A.O.; Ayo, C.K. Stock price prediction using the ARIMA model. In Proceedings of the 2014 UKSim-AMSS 16th International Conference on Computer Modelling and Simulation, Cambridge, UK, 26-28 March 2014; pp. 106-112.

9. Cortes, C.; Vapnik, V. Support vector machine. Mach. Learn. 1995, 20, 273-297. [CrossRef]

10. Fan, A.; Palaniswami, M. Stock selection using support vector machines. In Proceedings of the International Joint Conference on Neural Networks (IJCNN'01), Washington, DC, USA, 15-19 July 2001; Volume 3, pp. 1793-1798.

11. Cao, L.J.; Tay, F.E.H. Support vector machine with adaptive parameters in financial time series forecasting. IEEE Trans. Neural Netw. 2003, 14, 1506-1518. [CrossRef] [PubMed]

12. Nayak, R.K.; Mishra, D.; Rath, A.K. A Naïve SVM-KNN based stock market trend reversal analysis for Indian benchmark indices. Appl. Soft Comput. 2015, 35, 670-680. [CrossRef]

13. Zhang, X.D.; Li, A.; Pan, R. Stock trend prediction based on a new status box method and AdaBoost probabilistic support vector machine. Appl. Soft Comput. 2016, 49, 385-398. [CrossRef]

14. Patel, J.; Shah, S.; Thakkar, P.; Kotecha, K. Predicting stock market index using fusion of machine learning techniques. Expert Syst. Appl. 2015, 42, 2162-2172. [CrossRef]

15. Chen, K.; Zhou, Y.; Dai, F. A LSTM-based method for stock returns prediction: A case study of China stock market. In Proceedings of the 2015 IEEE International Conference on Big Data (Big Data), Santa Clara, CA, USA, 29 October-1 November 2015; pp. 2823-2824.

16. Hafezi, R.; Shahrabi, J.; Hadavandi, E. A bat-neural network multi-agent system (BNNMAS) for stock price prediction: Case study of DAX stock price. Appl. Soft Comput. 2015, 29, 196-210. [CrossRef]

17. Fischer, T.; Krauss, C. Deep learning with long short-term memory networks for financial market predictions. Eur. J. Oper. Res. 2018, 270, 654-669. [CrossRef]

18. Hochreiter, S.; Schmidhuber, J. Long short-term memory. Neural Comput. 1997, 9, 1735-1780. [CrossRef] [PubMed]

19. Sutskever, I.; Vinyals, O.; Le, Q.V. Sequence to sequence learning with neural networks. arXiv 2014, arXiv:1409.3215v3.

20. Yue-Hei Ng, J.; Hausknecht, M.; Vijayanarasimhan, S.; Vinyals, O.; Monga, R.; Toderici, G. Beyond short snippets: Deep networks for video classification. In Proceedings of the IEEE Conference on Computer Vision and Pattern Recognition, Boston, MA, USA, 7-12 June 2015; pp. 4694-4702.

21. Yümlü, S.; Gürgen, F.S.; Okay, N. A comparison of global, recurrent and smoothed-piecewise neural models for Istanbul stock exchange (ISE) prediction. Pattern Recognit. Lett. 2005, 26, 2093-2103. [CrossRef]

22. Selvin, S.; Vinayakumar, R.; Gopalakrishnan, E.A.; Menon, V.K.; Soman, K.P. Stock price prediction using LSTM, RNN and CNN-sliding window model. In Proceedings of the 2017 International Conference on Advances in Computing, Communications and Informatics (ICACCI), Udupi, India, 13-16 September 2017; pp. 1643-1647. 
23. Samarawickrama, A.J.P.; Fernando, T.G.I. A recurrent neural network approach in predicting daily stock prices an application to the Sri Lankan stock market. In Proceedings of the 2017 IEEE International Conference on Industrial and Information Systems (ICIIS), Peradeniya, Sri Lanka, 15-16 December 2017; pp. 1-6.

24. Siami-Namini, S.; Tavakoli, N.; Namin, A.S. A Comparison of ARIMA and LSTM in Forecasting Time Series. In Proceedings of the 2018 17th IEEE International Conference on Machine Learning and Applications (ICMLA), Orlando, FL, USA, 17-20 December 2018; pp. 1394-1401.

25. Rundo, F.; Trenta, F.; Di Stallo, A.; Battiato, S. Advanced Markov-Based Machine Learning Framework for Making Adaptive Trading System. Computation 2019, 7, 4. [CrossRef]

26. Rundo, F.; Trenta, F.; di Stallo, A.L.; Battiato, S. Grid Trading System Robot (GTSbot): A Novel Mathematical Algorithm for trading FX Market. Appl. Sci. 2019, 9, 1796. [CrossRef]

27. Hossain, A.; Nasser, M. Comparison of GARCH and neural network methods in financial time series prediction. In Proceedings of the 2008 11th International Conference on Computer and Information Technology, Khulna, Bangladesh, 24-27 December 2008; pp. 729-734.

28. Wijaya, Y.B.; Kom, S.; Napitupulu, T.A. Stock price prediction: Comparison of Arima and artificial neural network methods-An Indonesia Stock's Case. In Proceedings of the 2010 Second International Conference on Advances in Computing, Control, and Telecommunication Technologies, Jakarta, Indonesia, 2-3 December 2010; pp. 176-179.

29. Kara, Y.; Boyacioglu, M.A.; Baykan, Ö.K. Predicting direction of stock price index movement using artificial neural networks and support vector machines: The sample of the Istanbul Stock Exchange. Expert Syst. Appl. 2011, 38, 5311-5319. [CrossRef]

30. Adebiyi, A.A.; Adewumi, A.O.; Ayo, C.K. Comparison of ARIMA and artificial neural networks models for stock price prediction. J. Appl. Math. 2014, 2014, 614342. [CrossRef]

31. Patel, J.; Shah, S.; Thakkar, P.; Kotecha, K. Predicting stock and stock price index movement using trend deterministic data preparation and machine learning techniques. Expert Syst. Appl. 2015, 42, 259-268. [CrossRef]

32. Laboissiere, L.A.; Fernandes, R.A.; Lage, G.G. Maximum and minimum stock price forecasting of Brazilian power distribution companies based on artificial neural networks. Appl. Soft Comput. 2015, 35, 66-74. [CrossRef]

33. Li, Z.; Tam, V. A comparative study of a recurrent neural network and support vector machine for predicting price movements of stocks of different volatilites. In Proceedings of the 2017 IEEE Symposium Series on Computational Intelligence (SSCI), Honolulu, HI, USA, 27 November-1 December 2017; pp. 1-8.

34. Wang, J.H.; Leu, J.Y. Stock market trend prediction using ARIMA-based neural networks. In Proceedings of the International Conference on Neural Networks (ICNN'96), Washington, DC, USA, 3-6 June 1996; Volume 4, pp. 2160-2165.

35. Zhang, G.P. Time series forecasting using a hybrid ARIMA and neural network model. Neurocomputing 2003, 50, 159-175. [CrossRef]

36. Wang, Y.H. Nonlinear neural network forecasting model for stock index option price: Hybrid GJR-GARCH approach. Expert Syst. Appl. 2009, 36, 564-570. [CrossRef]

37. Lee, M.C. Using support vector machine with a hybrid feature selection method to the stock trend prediction. Expert Syst. Appl. 2009, 36, 10896-10904. [CrossRef]

38. Khashei, M.; Bijari, M.; Ardali, G.A.R. Improvement of auto-regressive integrated moving average models using fuzzy logic and artificial neural networks (ANNs). Neurocomputing 2009, 72, 956-967. [CrossRef]

39. Wang, J.J.; Wang, J.Z.; Zhang, Z.G.; Guo, S.P. Stock index forecasting based on a hybrid model. Omega 2012, 40, 758-766. [CrossRef]

40. Hadavandi, E.; Shavandi, H.; Ghanbari, A. Integration of genetic fuzzy systems and artificial neural networks for stock price forecasting. Knowl. Based Syst. 2010, 23, 800-808. [CrossRef]

41. Babu, C.N.; Reddy, B.E. A moving-average filter based hybrid ARIMA-ANN model for forecasting time series data. Appl. Soft Comput. 2014, 23, 27-38. [CrossRef]

42. Panigrahi, S.; Behera, H.S. A hybrid ETS-ANN model for time series forecasting. Eng. Appl. Artif. Intell. 2017, 66, 49-59. [CrossRef]

43. Kim, H.Y.; Won, C.H. Forecasting the volatility of stock price index: A hybrid model integrating LSTM with multiple GARCH-type models. Expert Syst. Appl. 2018, 103, 25-37. [CrossRef] 
44. Schumaker, R.P.; Chen, H. Textual analysis of stock market prediction using breaking financial news: The AZFin text system. In Proceedings of the ACM Transactions on Information Systems (TOIS), Honolulu, HI, USA, 8-12 March 2009; Volume 27, p. 12.

45. Pagolu, V.S.; Reddy, K.N.; Panda, G.; Majhi, B. Sentiment analysis of Twitter data for predicting stock market movements. In Proceedings of the 2016 International Conference on Signal Processing, Communication, Power and Embedded System (SCOPES), Paralakhemundi, India, 3-5 October 2016; pp. 1345-1350.

46. Mikolov, T.; Sutskever, I.; Chen, K.; Corrado, G.S.; Dean, J. Distributed representations of words and phrases and their compositionality. In Proceedings of the Advances in Neural Information Processing Systems, Lake Tahoe, NV, USA, 5-10 December 2013; pp. 3111-3119.

47. Daniel, J.; Martin, J.H. Speech and Language Processing. Computational Linguistics, and Speech Recognition; Prentice-Hall Inc.: Edinburgh, UK, 2000; pp. 22-105.

48. Lei, K.; Zhang, B.; Li, Y.; Yang, M.; Shen, Y. Time-driven feature-aware jointly deep reinforcement learning for financial signal representation and algorithmic trading. Expert Syst. Appl. 2020, 140, 112872. [CrossRef]

49. Rundo, F. Deep LSTM with Reinforcement Learning Layer for Financial Trend Prediction in FX High Frequency Trading Systems. Appl. Sci. 2019, 9, 4460. [CrossRef]

50. Halperin, I. The QLBS Q-Learner goes NuQLear: Fitted Q iteration, inverse RL, and option portfolios. Quant. Finance 2019, 19, 1543-1553. [CrossRef]

51. Baaquie, B.E. Quantum Finance; Cambridge University Press: Cambridge, UK, 2004.

52. Lee, R.S. Quantum Finance-Intelligent Financial Forecast and Program Trading Systems; Springer NATURE: Singapore, 2019.

53. Baaquie, B.E.; Du, X.; Tang, P.; Cao, Y. Pricing of range accrual swap in the quantum finance Libor Market Model. Phys. A Stat. Mech. Its Appl. 2014, 401, 182-200. [CrossRef]

54. Lee, R.S. Chaotic Type-2 Transient-Fuzzy Deep Neuro-Oscillatory Network (CT2TFDNN) for Worldwide Financial Prediction. IEEE Trans. Fuzzy Syst. 2019. [CrossRef]

55. Lee, R.S. COSMOS trader-Chaotic Neuro-oscillatory multiagent financial prediction and trading system. J. Finance Data Sci. 2019, 5, 61-82. [CrossRef]

56. Banna, G.L.; Camerini, A.; Bronte, G.; Anile, G.; Addeo, A.; Rundo, F.; Zanghi, G.; Lal, R.; Libra, M. Oral metronomic vinorelbine in advanced non-small cell lung cancer patients unfit for chemotherapy. Anticancer Res. 2018, 38, 3689-3697. [CrossRef]

57. Rundo, F.; Petralia, S.; Fallica, G.; Conoci, S. A nonlinear pattern recognition pipeline for PPG/ECG medical assessments. In CNS Sensors, Lecture Notes in Electrical Engineering; Springer: Cham, Switzerland, 2018; Volume 539, pp. 473-480.

58. Vinciguerra, V.; Ambra, E.; Maddiona, L.; Romeo, M.; Mazzillo, M.; Rundo, F.; Fallica, G.; di Pompeo, F.; Chiarelli, A.M.; Zappasodi, F.; et al. PPG/ECG Multisite Combo System Based on SiPM Technology. In CNS Sensors, Lecture Notes in Electrical Engineering; Springer: Cham, Switzerland, 2018; Volume 539, pp. 353-360. 